\title{
Short-time Fourier and wavelet transforms for fault detection in power transformers during impulse tests
}

\author{
L.Satish
}

Indexing terms: Fault detection, Short-time Fourier transform, Wavelet transform, Impulse tests, Power transformers, Time frequency analysis

\begin{abstract}
A novel approach using the short-time Fourier transform and wavelet transform (timefrequency analysis tools) for fault detection during impulse testing of power transformers is described. The neutral and/or capacitively transferred currents which are recorded during an impulse test can be directly analysed with this approach. These currents are considered to be evolving in time, i.e. as nonstationary signals, especially when there is a fault. Results from simulation studies are presented wherein the fault condition is modelled as a fast decaying transient superposed on the neutral current. A comparison of the two transforms is made to assess their ability to detect as small a fault as possible and other implemenational issues. Advantages of these methods over the conventional transferfunction method are demonstrated, and it appears that the wavelet transform is better suited for this task
\end{abstract}

\section{Introduction}

Impulse testing of power transformers is a routine to ascertain the integrity of insulation. This involves application of a specific number of predefined levels of impulse voltage and wave shapes. The resulting neutral and/or capacitively transferred currents are recorded. Standards stipulate comparison of these currents for detecting any visible variation in their shapes and, if none are present, the apparatus is adjudged as having passed the test. It is quite clear that this procedure is simple to adopt when the apparatus has a major fault resulting in large changes in shape of the pertinent currents, but is rather difficult when only a minor fault, say a sparkover between adjacent coils/turns lasting for a few microseconds occurs [1]. In such cases, the judgment is based on the expertise of the inspector and often becomes controversial. However, detection of such faults is very important and newer methods capable of accomplishing them are continually being explored.
With the availability of fast digital recorders and personal computers these waveforms are now being acquired and stored digitally, thus enabling their indepth analysis than mere visual examination of oscillographic traces. Initially, differences in waveforms were amplified and compared. Subsequently, development of the transfer function (TF) approach for fault diagnosis [2-4] was a milestone. The computed transfer functions at different voltage levels were compared and any deviation amongst them was considered to be due to a fault in the transformer. The main philosophy of this approach is that it is independent (at least theoretically speaking) of the shape of the applied voltage, chopping times, amplitude, bushing flashover and impulse-generator component faults, if any, which would not show up in the transfer function. But, contrary to this belief, practical experience in transformer testing has indicated some problems with regard to it being independent of the input excitation and chopping times [5]. The possible sources leading to errors and ambiguity in the transfer function comparison can be due to one or more of the following reasons:

(a) noise inherent in the acquired data, in spite of good shielding

(h) errors due to sampling, quantisation, A/D errors, finite record length

(cj different signal processing methods being adopted: windowing, filtering etc.

It is, perhaps that due to these many unanswered questions, this approach has not yet found its way into the relevant standards [6].

This paper presents simulation results of an entirely new approach, based on the time-frequency analysis of signals, for detecting faults from the neutral current waveforms. Of course, detection of major faults has never been an issue. Hence, the focus is on being able to detect accurately the smallest or minor type of faults, which are hard to detect using the TF approach.

\section{The underlying principle}

This approach is based on the principles of time-frequency analysis of signals, a fairly new signal processing tool [7]. Nonstationary signals (i.e. signals whose frequency varies with time) can be analysed with this technique. The primary reason for most real-world signals being nonstationary is that the production of Particular frequencies depends on the physical parameters and conditions of the system, which may change in time due to many reasons. It is important to be able to 
detect these changes accurately. There are a number of ways in which the input signal can be subjected to time-frequency analysis and, among them, the shorttime Fourier transform and wavelet transform are popular and, hence, have been considered here (Wigner distributions, Gabor transforms, bilinear transforms etc. have also been used for this purpose).

Time-frequency analysis of nonstationary signals indicates the time instants at which different frequency components of the signal come into reckoning. One direct consequence of such a treatment will be the possibility to accurately locate in time all abrupt changes in the signal and estimate their frequency components as well. It is in this very application that we are interested in here, i.e. to locate low-magnitude abrupt changes in the neutral current waveforms. In this context, we can visualise the neutral current as a nonstationary signal whose properties change or evolve in time, when there is a fault. This is particularly so when
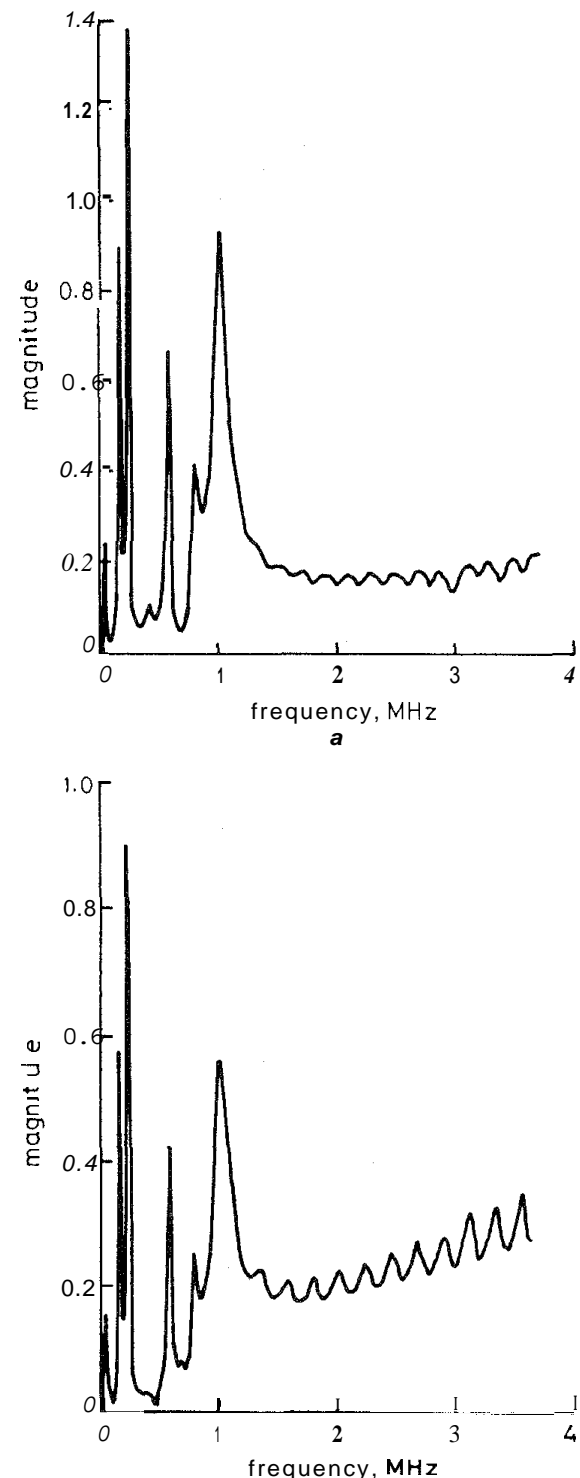

there is a momentary short-circuit between adjacent turns due to high stresses. This short-circuit lasts for a short interval only, say $1-2 \mu s$, and can be considered to manifest itself as a fast decaying oscillation superimposed on the neutral current. During such momentary short circuits, the circuit conditions are definitely altered, but due to the low magnitude and transient nature, the influence of such a short-circuit on the transfer function is almost insignificant, as will be shown later in the simulations.

\section{Brief introduction to short-time Fourier transform (STFT) [7]}

The primary objective of time-frequency analysis is to be able to define a function that will describe the energy density of a signal simultaneously in time and frequency, and is commonly used in applications to speech, sonar and acoustic signals. Among the few
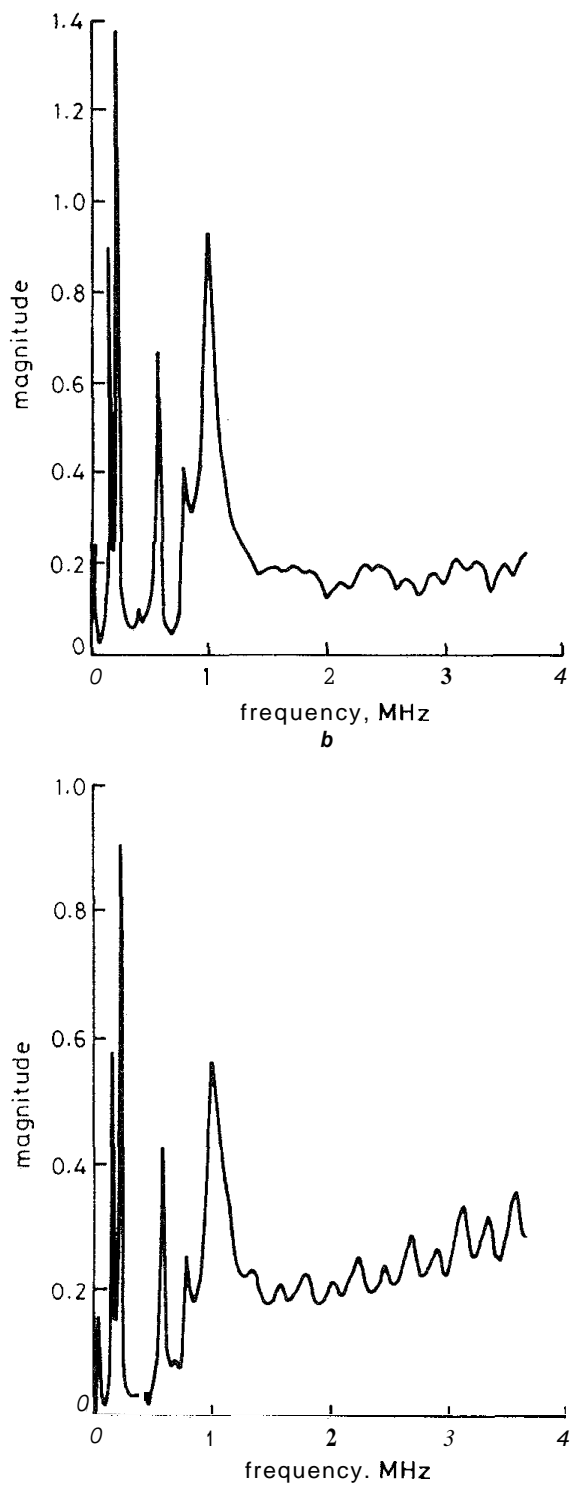

d

Fig.1 Effect o superposing faults on neutral currents on the transferfunction magnitude $a$ Case 1 , no fault 
tools, STFT happens to be very common and popular, because the concept behind it is simple yet powerful.

The basic idea of STFT is to slice up the signal into suitable overlapping time segments (using windowing methods) and then to Fourier analyse each slice to ascertain the frequencies contained in it. The accumulation of such spectra indicates how the spectrum is varying in time and is called the spectrogram. It is assumed that frequency information is associated with the time index in the middle of each slice of windowed data. STFT of a continuous-time signal $x(t)$ is defined as:

$$
\begin{aligned}
& \operatorname{STFT}(f, T)=\int x(t) w(t-T) \exp (-j 2 \pi f t) d t \\
& -\infty<t<\infty
\end{aligned}
$$

where $w(t)$ is the window function whose position is translated in time by $\tau$. There are some limitations associated with STFT, the first being the window length. It is obvious that a wide window yields a good
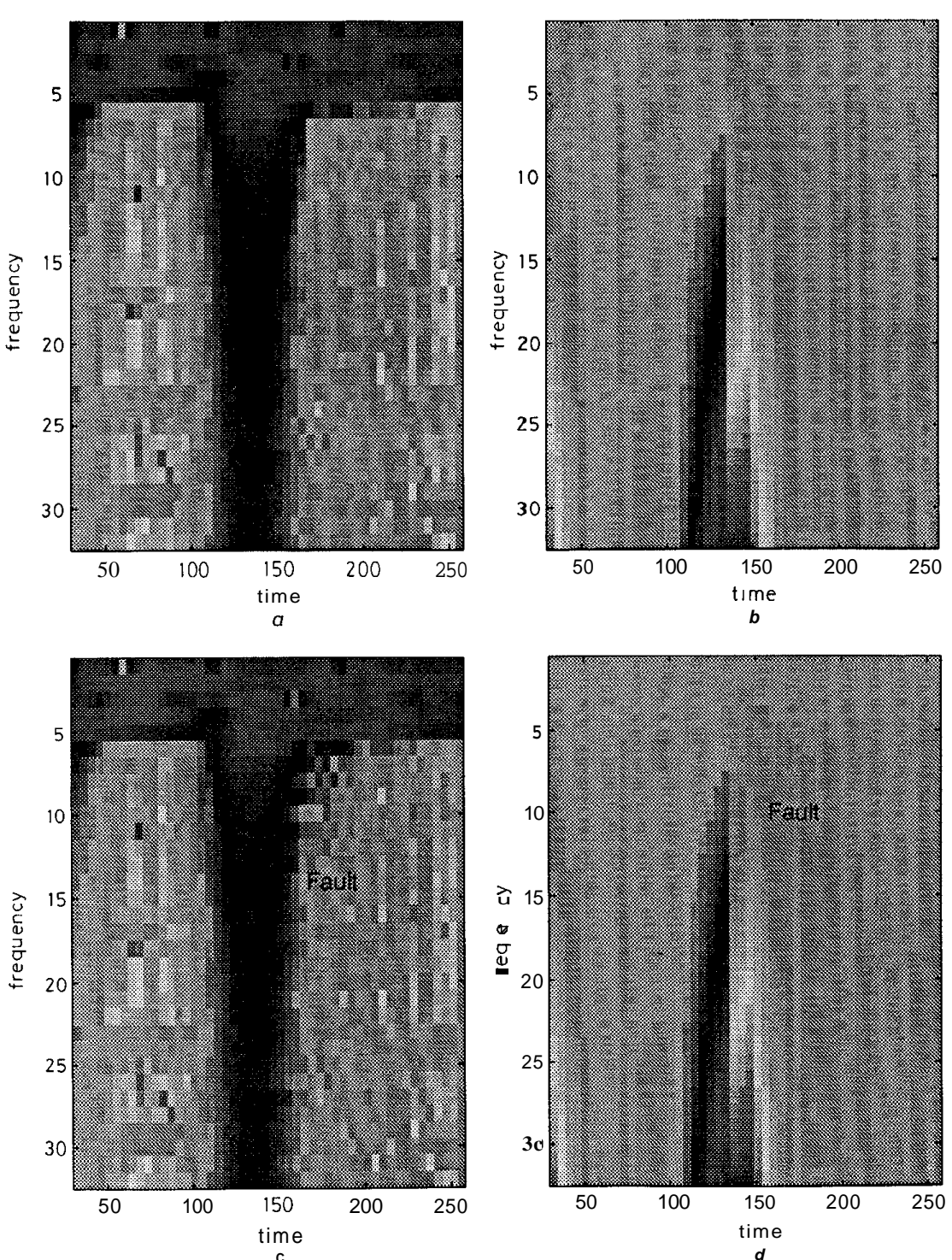

Fig.2, STFT magnitude and phase for case I with window length $=64$ $a$ Magnitude, no fault $b$ Phase, no fault c Magnitude, with fault $d$ Phase. with fault

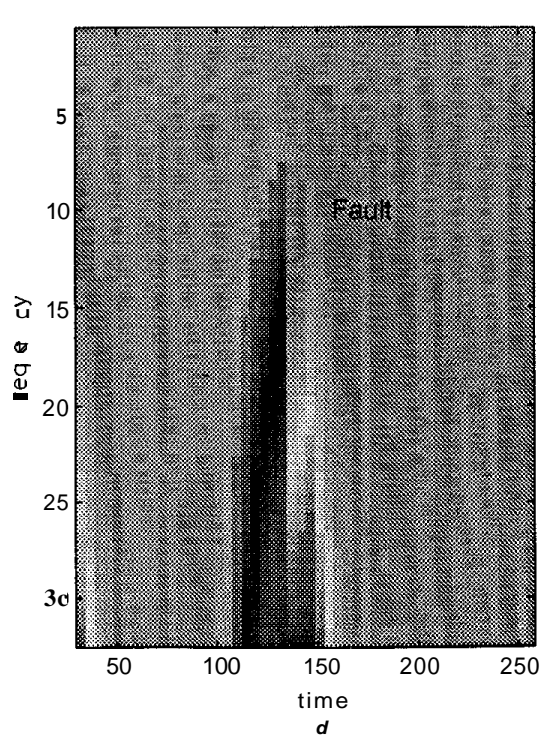

resolution in the frequency domain, but poor resolution in the time domain, and vice versa. So, in practical situations, a compromise between the two resolutions has to be made. Secondly, raw STFT is computationally expensive, but ways of accelerating it by avoiding redundant calculations are available in the literature. These drawbacks notwithstanding, STFT is an ideal tool in many respects, the most important being its excellent spectrogram structure, which is consistent with our intuition regarding frequency spectra, thereby qualifying as a good visualisation tool.

\section{Brief introduction to wavelet transforms (WT) $[8,9]$}

Signal processing using wavelet theory has emerged as a powerful tool over the past ten years and has led to significant developments in data analysis, data compression, image and speech processing, multiresolution 
analysis etc. The WT like the Fourier transform decomposes a given signal into its frequency components, but differs in providing a nonuniform division of the frequency domain. In addition, unlike the Fourier transform which gives a global representation of the signal, WT provides a local representation in both time and frequency. This results from the fact that the analysing basis functions in the case of the Fourier transform (namely sines and cosines) extend over infinite time, whereas they are compactly supported functions in the case of WT, thus giving them the localisation property. This property greatly facilitates analysis of nonstationary signals, transient detection etc. A mathematical definition of WT follows:

Let $x(t)$ denote a continuous-time finite energy signal, then WT of $x(t)$ is defined as:

$$
\mathrm{WT}(a, b)=\int x(t) g_{(a, b)}(t) d t \quad-\infty<t<\infty
$$
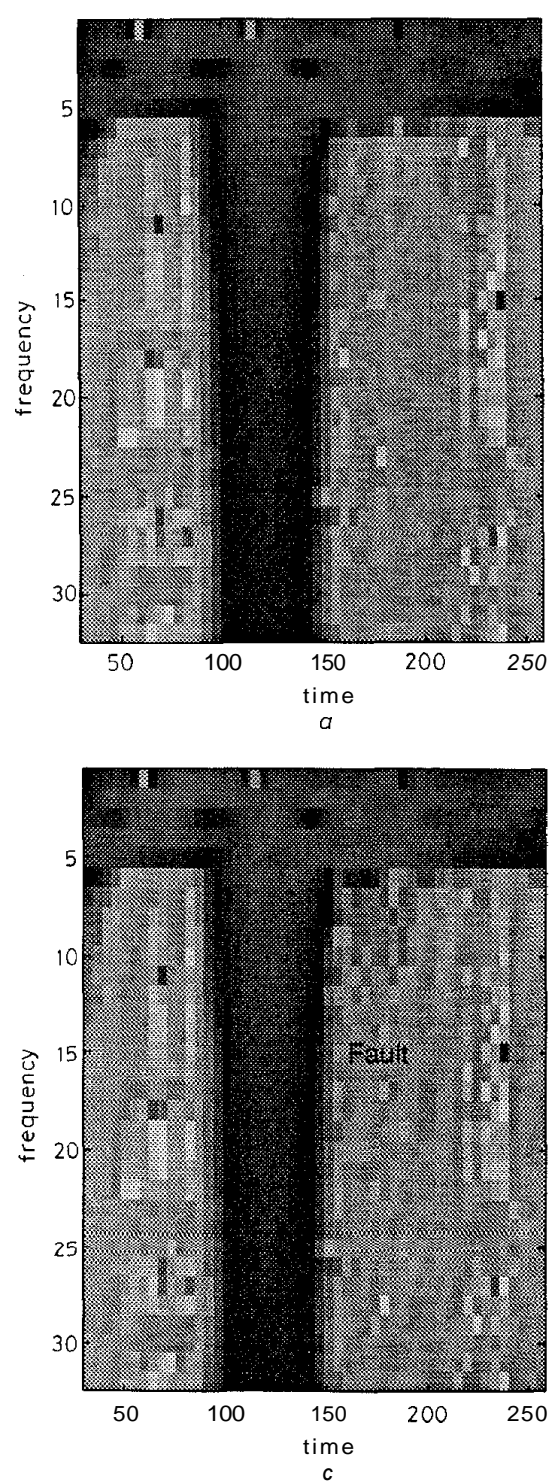

Fig.3 STFT magnitude and phase for case 2 with window length $=64$ a Magnitude, no fault $a$ Phase, no fault c Magnitude, with fault c Phase, with fault where

$$
g_{(a, b)}(t)=|a|^{(-1 / 2)} g((t-b) / a)
$$

is called the base function or mother wavelet. $a, b$ (real, $a \neq 0)$ are the dilation and translation parameters, respectively. A restriction on the choice of $g(t)$ is that it must have a zero average value and be of short duration, which, mathematically, is called the admissibility condition on $g(t)$. Daubechies' wavelet, Morlet wavelet, Harr wavelet are some examples of popularly used functions for $g(t)[8,9]$.

In general, STFT and WT may be interpreted as inner products of the signal and a set of analysing functions located throughout in the time-frequency and time-scale planes, respectively. The function WT $(a$, $b$ ) gives an idea of the contributions to the signal around time $b$, at a scale $a$ and hence leads to a timescale decomposition when compared to the time-fre-
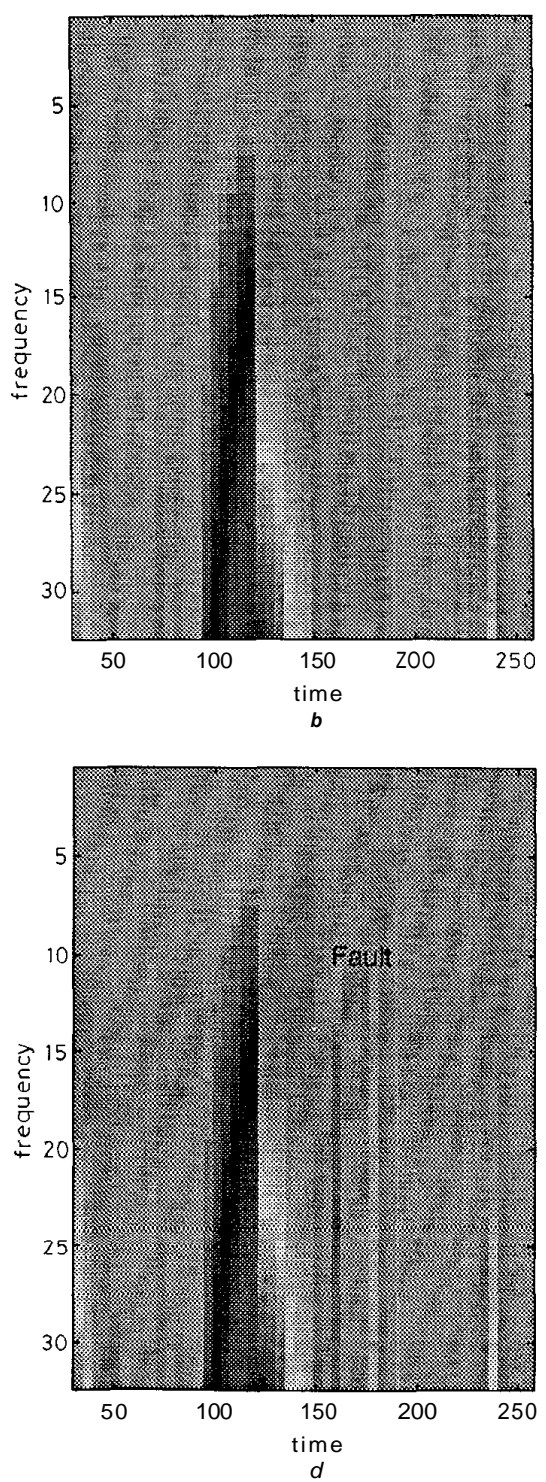
quency decomposition obtained from STFT. The quantities frequency and scale are inversely related, i.e. a small value of $a$ implies a high frequency and vice versa. Time-scale decomposition obtained from the WT is referred to as a scalogram. Among the many wavelet functions, the Morlet wavelet

$$
g(t)=\exp \left(-j \omega_{0} t\right) \exp \left(-t^{2} / 2\right)
$$

has been shown to yield the best time-frequency localisation [10], and, hence, has been chosen here. Briefly, the steps involved in computing scalograms are given by the following pseudocode:

Input: sampling rate, minimum, maximum and stepsize of scale-factor $a$, input signal

Do Loop scale-factor $=$ minimum, maximum, stepsize:

for each scale-factor
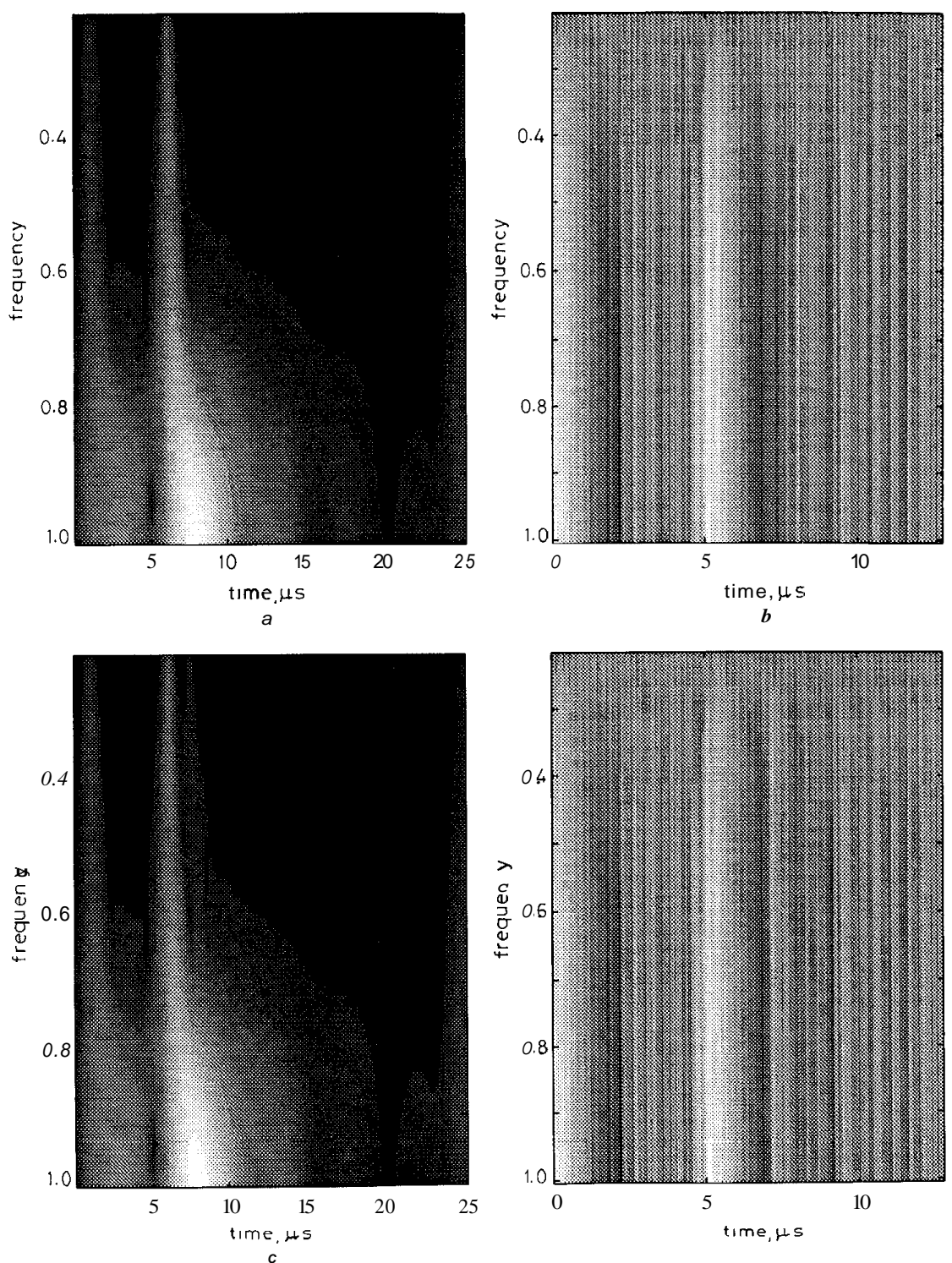

compute length, 1w, of Morlet wavelet required compute Morlet wavelet sequence with $b=0$ convolve this with the input signal accumulate convolution result after discarding initial and final $1 \mathrm{w} / 2$ points

\section{End Loop}

Plot magnitude and phase of accumulated results to yield scalograms

(Note: For computing the Morlet wavelet, a value of -6 to +6 time units is used because its values are negligibly small for $A b s(t)>6$ time units. Based on this and the sampling rate, $l w$ is obtained, using which a linear time-space is generated for computing the Morlet wavelet sequence. Anonymous FTP sites for downloading useful wavelet transform and related programs are: playfair.stanford.edu in /pub/wavelab, ftp.tsc.uvigo.es in /pub/Uvi_Wave/matlab/)

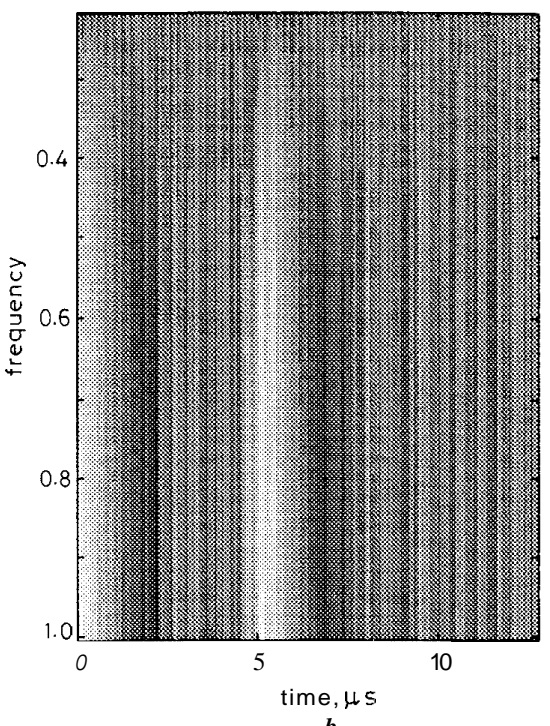

b

Fig.4 Scalogram magnitude and phase for case I

a Magnitude, no fault

$b$ Phase, no fault

c Magnitude, with fault

$d$ Phase, with fault 


\section{Simulation}

In this work, two neutral currents (case 1 and case 2) corresponding to two chopped voltages (4096 points, sampled at 50ns) with different chopping times are used [11]. Owing to the chopped wave and its consequences, the transformer insulation is subjected to very high stresses which are usually the cause of failures. Failure detection under these conditions is a good index of any method. Also, it has been well established that initial effects of the fault manifests itself in the neutral current with a delay of about $1-2 \mu$ s after the instant of chopping [1]. This situation is simulated by superposing a short-duration (exponentially decaying) oscillating transient onto the neutral current, starting at time instant $t=6.5 \mu \mathrm{s}$. Its frequency, decay time constant and amplitude are chosen as $2 \mathrm{MHz}, 0.25 \mu \mathrm{s}$ and about $6 \%$ of the neutral current peak, respectively.
Addition of the fault component does not produce any appreciable observable change in the neutral current as can be seen in a later Figure. To see the possible impact of proximity of chopping time and occurrence of fault, different chopping times, viz. two times to chop $5 \mu$ s and $4.4 \mu$ s (case 1 and case 2 , respectively), were chosen. The goal is to detect as small a change as possible under these circumstances.

\section{Results and discussion}

First, the ability of the transfer function approach to such superposed faults is investigated. Transfer functions calculated with and without superposition of fault are shown in Fig. 1, for case 1 and case 2. As can be seen, owing to the inclusion of the fault, there are only small variations in the transfer function, but its effect is global, i.e. spread throughout the spectrum. Therefore,
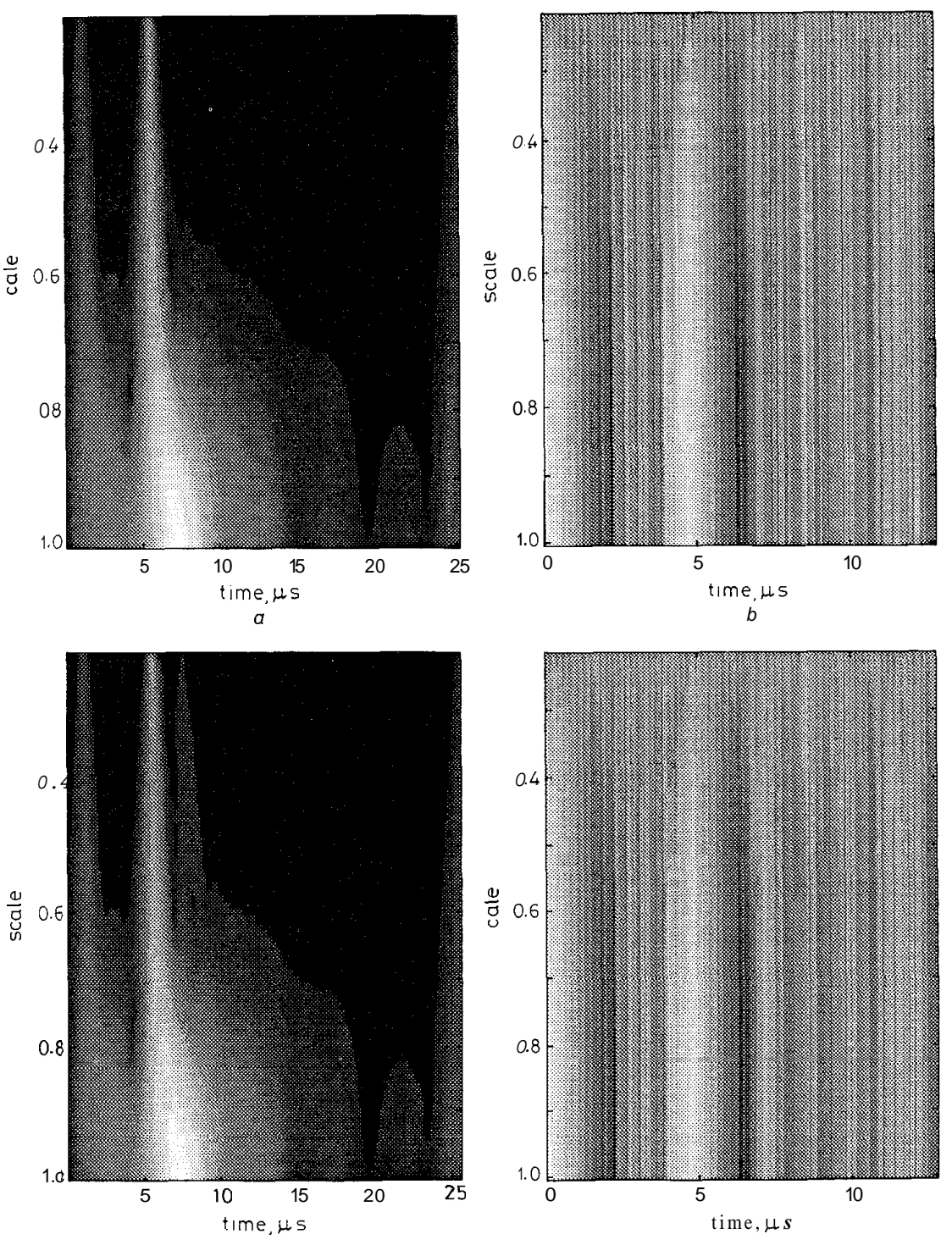

Fig.5 Scalogram magnitude and phase for case 2

a Magnitude, no fault

$b$ Phase, no fault

$c$ Magnitude, with fault

$d$ Phase, with fault 
neither the frequency content of fault nor the time of its occurrence can be determined using the $\mathrm{TF}$ approach. During simulations, it has been observed that, when the frequency of the superposed fault coincides with one of the poles of the system, even this small variation is not apparent. Hence, such small disturbances are difficult, if not impossible, to resolve by the transfer function approach.

As already mentioned in the STFT approach, choice of window length plays an important role. Various window lengths have been tried out. As locating the time instant of fault occurrence is important, a narrow window of length 64 and 32 points were found suitable, even though the frequency resolution was poor. A Hamming window translated each time to the right by 4 data points was used. As any phenomenon in the neighbourhood of the chopping time is of interest, only the first 1024 points were processed. Such a selective segmentation of the time records is not possible with the transfer function approach. Spectrogram magnitude and phase plots for case 1 and case 2 are shown in Figs. 2 and 3, respectively. The dark patch parallel to the frequency axis is due to the high-frequency components in the neutral current corresponding to the chopping action. The occurrence of the fault can be visualised with some effort in both the magnitude and phase plots, just after the chopping time. A colour plot will definitely be of more help. Using a narrower window, as expected, yields an improved time localisation of the fault. Higher-magnitude faults can be visualised more easily.

The ability of the wavelet transform approach to detect faults is investigated next. The first 512 points of the neutral current record are taken for the purpose of analysis. The scale factor $a$ is varied from 0.22 to 1.0 in steps of 0.01 (corresponding to a frequency range of $4.5 \mathrm{MHz}$ to $1 \mathrm{MHz}$ ). Smaller step sizes will lead to finer resolution of the scalograms, which of course will con-
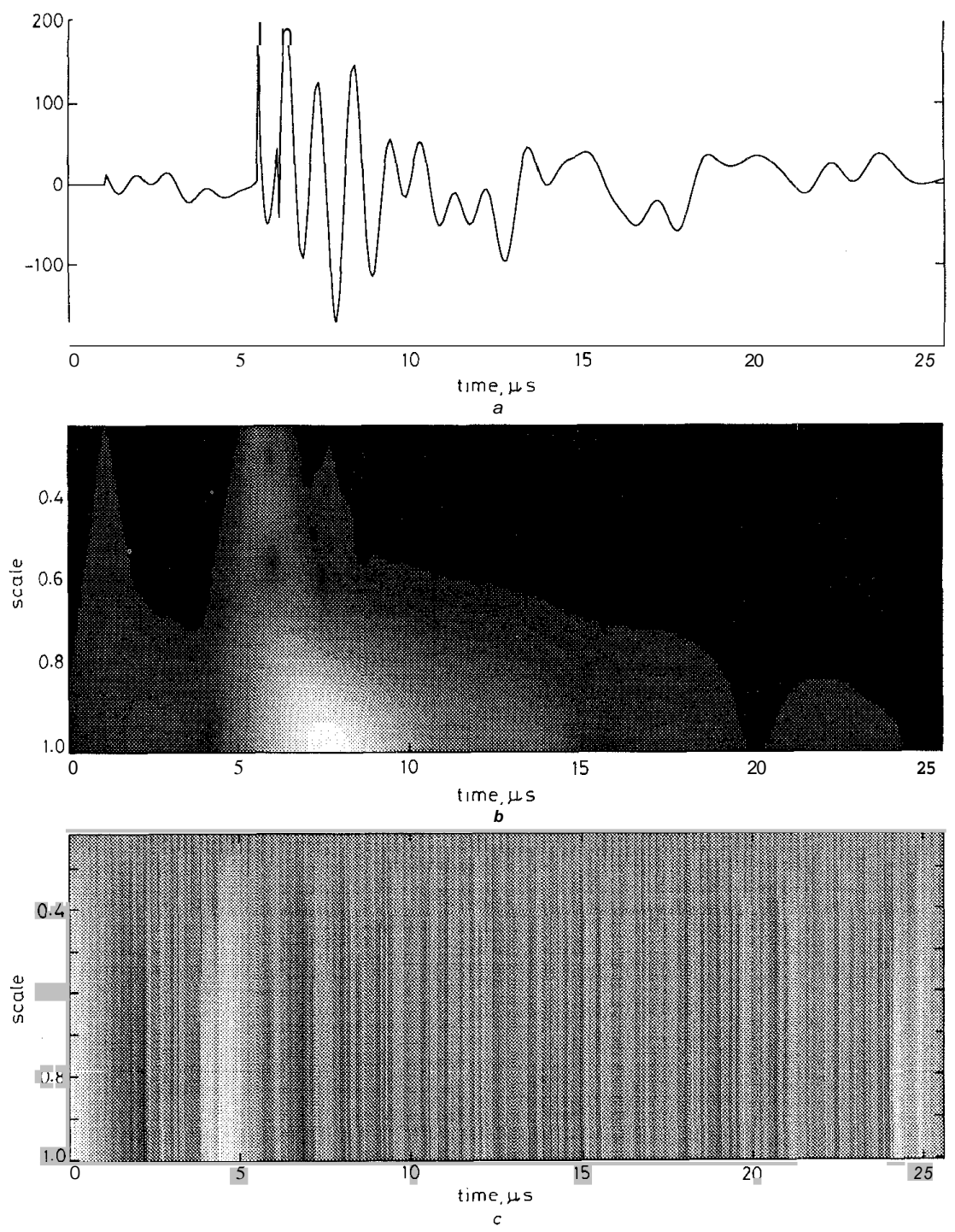

Fig.6 Scalogram for the difference between case 1 and case 2 with fault superposed on case 1

a Case 1 - case 2

$b$ Magnitude

c Phase 
sume more computation time. If a low-frequency phenomenon has to be investigated, then values of $a$ greater than 1.0 are to be chosen. Scalogram magnitude and phase plots for the two cases are plotted in Figs. 4 and 5, respectively. Visualisation of the fault and the time instant of its occurrence are very clearly and accurately detectable in these plots. In addition, the initial delay of $1 \mu$ s can also be seen in the magnitude plots. High-resolution colour monitors and plots are recommended for improving visualisation of the results. Occurrence of a fault can be inferred from these plots, whenever a sharp protrusion-like structure is present. The magnitude plot in Fig. $4 a$ shows two such sharp protrusion-like structures within the first $10 \mu \mathrm{s}$, with the first one at $1 \mu \mathrm{s}$ corresponding to the initial delay followed by the other due to the chopping phenomenon. When the fault is superposed, an additional protrusion appears (see Fig. 4c) corresponding to the fault. The time instant and frequency content of the fault component are more easily discernable in this case. Similar results are obtained for case 2 as well (Fig. 5). In all cases, unwrapped phase angle has been shown. It is a fact that phase plots are difficult to interpret, more so when the input signals are complex (highly oscillating), as in the present case. Only a careful comparison of phase plots (with and without fault superposed) indicates changes. Thus, when compared to STFT, the wavelet transform yields results from which faults are clearly distinguished and are easy to interpret.

Another possible approach, when given two neutral current records with slightly different chopping times and one of the records having a superposed fault, would be to subtract the two currents and thereafter compute the scalogram. Scalogram plots along with the difference current are shown in Fig. 6. Here, the magnitude and time instant at which the transient is superposed is the same as in the previous cases. We see that there are four such sharp protrusion-like structures (within the first $10 \mu \mathrm{s}$ ) and it is easy to interpret them. The first one matches with the beginning of the signal. The next two of them (very close to each other) are due to the two different chopping times, and the last one is due to the fault. The ability to distinguish from the scalogram the two chopping instants followed by a fault is illustrated.

Detection of small or minor faults, particularly during chopped voltage tests is still considered as a difficult task, even in the TF approach. It is worth mentioning here that the proposed approach processes the neutral current directly, as in the conventional way. It only acts as an additional visualisation tool for the test engineer. The ability to detect unambiguously such low-amplitude fault-initiated transients directly from the neutral current records, with sufficient accuracy in time and acceptable accuracy in frequency simultane- ously, is the great advantage of this approach over the prevalent transfer function methods. Also, the possibility to selectively process any length of the signal record from the acquired data is unique to this method. However, this paper only describes, perhaps, the first attempt that is found to be robust and presumably better suited for the task of fault diagnosis. Further investigations have to be carried out to verify its applicability for practical data from various impulse tests.

\section{Conclusions}

A new approach based on time-frequency analysis of signals has been proposed, for analysing the neutral currents directly, for fault diagnosis during impulse tests on power transformers. Two popular time-frequency tools, the short-time Fourier transform and wavelet transform were investigated for this purpose. Preliminary simulation results presented highlight their advantages over the conventional transfer function approach, and go on to indicate its potential and suitability as a possible future tool for fault detection.

\section{Acknowledgment}

The author expresses his thanks to Prof. Gururaj (Private Consultant, formerly with the Indian Institute of Science, Bangalore) for making available basic data used in the simulations. His critical evaluation of the manuscript and discussions which the author had with him on issues of the proposed approaches and fault diagnosis were of great help.

\section{$9 \quad$ References}

1 HICKLING, G.H.: 'High voltage impulse testing of transformers, Part I and 11', Electr. Rev., 1956, 12, pp. 1-14

2 MALEWSKI, R., and POULIN, B.: 'Impulse testing of power transformers using transfer function method', IEEE Trans. Power Deliv., $1988,3,(2)$, pp. 476489

3 MALEWSKI, R. CLAUDI, A GOCKENBACH, E., MAIER, R., and FELLMANN, K.H.: 'Five years of monitoring the impulse test of power transformers with digital recorders and the transfer function method'. Paper 12-201, CIGRE, 1992

4 LEIBFRIED, T., and FESER, K.: 'Off-line and on-line monitoring of power transformers using the transfer function method'. Proceedings of IEEE Int. Conf. Elect. Insul., Montreal, June 1996, pp. 34

5 MULLER, W., and STEIN, W.W.: 'Discussions Group 12'. CIGRE, 1992, Vol. 1, pp. 23-25

6 General Report for Group 12, CIGRE 1992, Vol. 1

7 COHEN, L.: 'Time-frequency analysis' (Prentice-Hall Inc., New Jersey, 1995)

8 RIOUL, O., and VETTERLI, M. 'Wavelets and signal processing', IEEE Signal Process. Mug., October 1991, 8, (4), pp. 14-38

9 MEYER, Y. (Ed.): 'Wavelets and applications'. Proceedings of the International Conference, Marseille, France, May 1989, (Masson and Springer Verlag)

10 UNSER, M., and ALDROUBI, A. 'A review of wavelets in biomedical applications', Proc. IEEE, 1996, 84, (4)

11 GURURAJ, B.I.: Private communications 